\title{
EL CÁNTICO DE LAS CRIATURAS DE FRANCISCO DE ASÍS: UN APORTE A LA HISTORIA DE LA LENGUA ITALIANA DEL DUECENTO
}

\author{
Marcos Viquez Ruiz
}

\begin{abstract}
RESUMEN
Este artículo presenta el aporte de Francisco de Asís a la historia de la lengua italiana del Duecento, sobre la base del Cántico de las Criaturas o Cántico del Hermano Sol (1224), pues el poeta franciscano escribió el Cántico en romance vulgar umbro, y no en latín, como lo establecía la norma en el siglo XIII.
\end{abstract}

\begin{abstract}
This article presents the contribution of Francis of Assisi to the history of the Italian language of the Duecento, on the basis of the Chant of the Creatures or The Chant of the Brother Sun (1224), for the poet wrote the Chant in vulgar Umbrian and not in latin, the norm established in the XIII century.
\end{abstract}

\section{Introducción}

Francisco de Asís escribió varias obras en latín, las cuales contienen instrucciones o reglas a sus seguidores; pero sobre todo, entre los textos líricos más antiguos de la literatura italiana sobresale el famoso Cantico delle Creature o Cantico di Frate Sole, que en adelante denominaremos para efectos prácticos Cántico de las Criaturas, escrito por él en romance vulgar umbro, y no en latín, como era la norma en el siglo XIII.

Sotto il nome di San Francesco è giunto fino a noi un cantico in volgare umbro, dettato in una sorta di prosa ritmica e rimata, che, nella divisione irregolare dei versetti, sembra riecheggiare le forme della liturgia e non trova corrispondenza nella letteratura italiana contemporanea (Sapegno 1948: 11, el destacado es nuestro).

En la Biblioteca Comunal de Asís se conserva un manuscrito misceláneo del siglo XIII o del siglo XIV, el cual pone de maniesto que el motivo religioso propio de la Edad Media caracterizó la primera literatura italiana. 
Dentro de este contexto histórico, el Cántico de las Criaturas se convierte en un hermoso himno que ensalza a todas las criaturas hermanas (el sol, el agua, el viento, el fuego, la muerte) y por este motivo amadas y glorificadas en Dios "Altissimu, onnipotente, bon Signore".

El presente artículo no pretende, de ninguna manera, la realización de un análisis teológico del proyecto evangélico de Francisco de Asís. Antes bien, el objetivo principal de nuestro estudio es presentar su aporte a la historia de la lengua italiana del Duecento, sobre la base del Cántico de las Criaturas.

Sin embargo, desde el punto de vista histórico sería injusto omitir que estamos en presencia de una de las figuras más destacadas de la cristiandad, pues en Italia, el Duecento fue, ante todo, un período de apogeo y de reafirmación religiosa.

Al respecto, Thaddée Matura afirma que:

Francisco de Asís no es solamente una de las figuras más grandes de la humanidad y del cristianismo, sino también el iniciador de un género de vida según la forma del santo Evangelio, pues, de acuerdo con el mismo autor, "el movimiento lanzado cuestionaba igualmente muchas de las estructuras de la Iglesia" (1978: 31).

Por esta razón, en el presente trabajo también se encontrarán algunas referencias sobre el contenido religioso de su obra.

\section{Vida de Francisco de Asís (1182-1226)}

Patrono principal de Italia y patrono universal de la Ecología, San Francisco ${ }^{1}$, nació en Asís, ciudad italiana de Umbría, en el seno de una familia burguesa. Se le bautizó con el nombre de Juan, pero su padre, Pedro Bernardone, un rico comerciante, lo llamó Francisco en honor de Francia, país natal de su esposa, doña Pica.

Battezzato col nome di Giovanni, fu chiamato Francesco dal padre, Pietro di Bernardone, ricco mercante di panni, in ricordo dell'origine francese della moglie, madonna Pica. Francesco appartiene a una famiglia borghese benestante, apprende tre lingue: il francese, il latino e il volgare (Ricciardi 1989: 50).

Al principio ejerció la profesión de su padre, y se dedicó a vivir la juventud propia de un despreocupado burgués, participando del entorno familiar y social.

Al estallar la guerra entre las ciudades de Asís y Perusa, Francisco tomó las armas en salvaguardia de su ciudad y cayó prisionero, en 1202, en manos del enemigo.

Un año después, puesto en libertad, regresó a Asís. En 1206, se sintió llamado por Dios, razón por la que tomó la decisión de abandonarlo todo por Cristo, incluida su propia familia.

Optó por la pobreza absoluta, curó a los leprosos y se entregó al apostolado; con el pequeño grupo de sus primeros discípulos: Bernardo de Quintavalle, Pedro Catáneo, Egidio, Gil Tancredi, Maseo y León, en 1209 constituyó su orden. Su ejemplo y su palabra conquistaron pronto nuevos seguidores; con Clara Scifi (Santa Clara), fundó en 1212 la segunda orden (religiosas), y en 1221, la tercera orden, integrada por seglares (mujeres y hombres), con lo que el movimiento franciscano se extiende a toda la sociedad. 
Francisco de Asís falleció el 4 de octubre de 1226. La voz del pueblo lo canonizó en vida. El Papa Gregorio XI no hizo más que sancionar su veredicto el 16 de julio de 1228 , a menos de dos años de su muerte.

Además de modelo de vida, ha sido incluido por los estudiosos y críticos de la literatura italiana, como poeta religioso importante del Duecento. No en vano, Dante Alighieri elogia a San Francisco en La Divina Comedia, en El Paraíso, Canto XI.

\begin{abstract}
(...) Mas para no continuar en estilo demasiado oscuro, reconoce en mis difusas palabras a los amantes Francisco y Pobreza. (...) Y cuando a Aquel que tanto le había ensalzado plugo llevarlo a lo alto, a la recompensa que mereció por hacerse tan humilde, recomendó a sus hermanos, como a legítimos herederos, su más amada dama y les ordenó que la amaran fielmente; y luego su alma preclara se desprendió del mortal seno para volver a su reino, y no quiso para su cuerpo otra tumba que la tierra (Dante 1992: 349-50).
\end{abstract}

Con el propósito de situar al poeta franciscano dentro de su época, haremos un brevísimo recorrido histórico-cultural por la Edad Media.

\title{
2. Características histórico-culturales de la Edad Media
}

La mayoría de los historiadores coinciden en que la Edad Media se sitúa entre la Antigüedad Clásica y el Renacimiento, comprendiendo diez siglos: del V al XV.

Más exactamente, la Edad Media coincide con la era de expansión del Cristianismo en el mundo y va, desde la caída del Imperio Romano, con la invasión de los bárbaros del Norte (godos) y los de Oriente (árabes), hasta la época de los grandes logros del siglo XV: la invención de la imprenta, el encuentro de las Culturas en el Continente Americano, el nuevo enfoque de la filosofía, la literatura y el arte renacentistas.

Sin embargo, Quirós Rodríguez (1997: 83) afirma que:

Con una dosis de mala intención, tal edad ha sido colocada en medio de las otras dos: la Antigua, cuando Grecia y Roma habían afirmado al hombre: y el Humanismo-Renacimiento, cuando el hombre es reafirmado según sus cualidades y virtudes terrenales, sobre todo, por el predominio de la razón.

Más adelante agrega:

La Edad Media no está conformada por diez siglos, en el transcurso de los cuales el ser humano estuvo allí meramente parado, inactivo, pasivo (...) esperando a que todo le lloviera del cielo. Fue una edad convulsiva, penosa, de creaciones (...) ¿de barbarismo, tinieblas, errores y horrores de toda especie? Afirmar y aceptar únicamente esto demuestra falta de conocimiento, tanto histórico como filológico (1977: 166).

Entre otros aspectos, la investigación realizada por el mismo estudioso del latín y de las lenguas romances (1997: 23-4) rescata lo positivo de la Edad Media, pues en esta época se fundan distintas universidades que, para el caso de Italia, cronológicamente son las siguientes: Salerno (¿Siglos VIII, IX?); Boloña (1088); Reggio (1188 ó 1210); Vicenza (1204); Arezzo (1215); Padua (1222); Nápoles (1224), Vercelli (1228); Piacencia (1248); Siena (1357); 
Treviso (1263) Roma (1303); Perusa, (1308); Pisa (1343); Florencia, (1349); Pavía (1361 ó 1389); Ferrara (1381); Turín (1405); Catania (1444).

Por lo demás, para Isaac Felipe Azofeifa (1984: 79), la Edad Media culmina en el siglo XIII, ya que es durante este siglo cuando se recogen los frutos intelectuales y artísticos de un largo proceso que dio inicio con la concepción del mundo de la cultura greco-romana, seguida luego por el ideal cristiano de vida y la vitalidad germana.

Dentro de estas concepciones del mundo de finales de la Edad Media, emerge, pues, la figura de Francisco de Asís, movido por este "ideal cristiano de vida".

Siguiendo con la temática del presente artículo, nos referiremos someramente a la relación entre el latín y las lenguas romances o neolatinas.

\title{
3. El latín y las lenguas romances
}

Al igual que en el resto de las naciones neolatinas, en Italia, la formación de la lengua romance se desarrolló lentamente, con una diversificación dialectal preliminar a la unidad lingüísica de la lengua italiana. Al derrumbarse el Imperio Romano, en todos los territorios unificados culturalmente por Roma surgieron diferencias lingüísticas, que dieron lugar a las distintas lenguas romances, románicas o neolatinas.

\begin{abstract}
La cuestión referente al momento en que surgen las lenguas romances, (...) ocurrió en el momento en que la fractura de la unidad del latín fue un hecho y en que se dieron cuenta de su diferenciación, esto es, entre el año 476 y el siglo XI D. C. Los vulgarismos o romanismos, que como hemos visto estaban siempre presentes desde tiempos remotos en el latín hablado, pudieron desarrollarse libremente solo después de 476, fecha de la caída del Imperio (Vidos 1977: 261, el destacado es nuestro).
\end{abstract}

Por lo demás, así como en las regiones de Francia situadas aproximadamente al sur del río Loira se formó la "langue d'oc" (partícula afirmativa "oui"), y en aquellas situadas al norte del mismo río, se forjó la "langue d'oïl (partícula afirmativa "oui"); en Castilla surge el castellano, y, en Italia, el italiano.

Entre las lenguas románicas, la italiana viene a representar la continuación central o directa del tronco latino, al paso que el portugués, el español, el catalán, el provenzal, el francés y el rumano vienen a ser más bien sus ramificaciones (Vossler 1951: 11).

En los distintos territorios de la Península, el latín cedió su lugar a los dialectos itálicos, es decir, a las variedades locales de un latín popular bastante desarrollado. De dichos dialectos, ya más lejanos del latín, emergió la lengua italiana como resultado de la misma unificación que se dio también en otras naciones tales como España o Francia.

Sin embargo, para los estudiosos romanistas, el italiano se consolidó tardíamente porque el latín se mantenía vivo al haberlo adoptado la Iglesia y también porque representaba la tradición cultural.

Por ambas razones, la mayor parte de las obras escritas en Italia durante el siglo XIII lo están en latín, como otras del siglo XIV. Contrariamente, la lengua del pueblo, denominada el "volgare", fue motivo de escasas producciones literarias. 
Los italianos, ni aun en la más remota época de la Edad Media, no han dejado nunca de sentirse descendientes de los antiguos romanos. El conocimiento y el uso del latín fue en la península italiana más fácil y más extendido que en cualquier otro país, de suerte que para la escritura no se empleó la lengua popular hasta época tardía (Vossler 1951: 17).

En la Biblioteca de Cassino, se encuentra el Placito de Capua o Plácito Capuano, primer documento oficial de la historia en "volgare italiano". Se trata de un pergamino notarial del año de 960, que contiene cuatro veces la misma fórmula de juramento pronunciado por cuatro testigos reconociendo el derecho del Monasterio de Montecassino sobre algunas tierras. Dicha fórmula, precedida por partes explicativas escritas en latín, dice así en dialecto campano (Capua):

"Sao ko kelle terre, per kelle fini que ki contene,

trenta anni le possette parte Sancti Benedicti”.

Es reconocido también como el documento más antiguo escrito en "volgare italiano" el Indovinello veronese (Adivinanza veronesa). Al menos la primera parte de este documento está redactada en romance, y la segunda en latín eclesiástico medieval.

A continuación, transcribimos la edición paleográfica en "volgare italiano":

1. "separebabouesalbaprataliaarabat \& albouersorioteneba \& negrosemen

2. seminaba".

Para Moreno y Peira (1979: 403):

Aún hoy se sigue discutiendo si el autor pretendió escribir en latín y fue incapaz de eliminar los vulgarismos, o bien su intención era escribir en un italiano que aún estaría muy apegado al latín.

En cuanto al "volgare umbro", en el que Francisco de Asís escribió el Cántico de las Criaturas, se hablaba entre el Tíber y el Nera en la antigua Umbría. Era el más septentrional de los dialectos itálicos y el que mejor se conoce, gracias a las conocidas Tablas Iguvinas, siete tablas de bronce, escritas por las dos caras, descubiertas en Gubbio en 1444.

En lo que a este artículo corresponde, es importante reafirmar que el Cántico de las Criaturas de Francisco de Asís es el primer texto literario italiano escrito enteramente en "volgare umbro".

Son Cantique du frère Soleil (Cantico di Frate Sole) ou Cantique des Créatures (1224) est le premier grand poème de la langue italienne (dialecte ombrien) (Robert 1984: 676, el destacado es nuestro).

\section{Las hipótesis de los astrónomos}

A manera de ilustración, y con el deseo de entrelazar el tema del "Canticum Fratis Solis" con el despunte del alba del Renacimiento italiano, se hará una brevísima mención sobre las hipótesis formuladas por connotados astrónomos que enfocaron su mirada hacia el firmamento. 


\subsection{Nicolás Copérnico (Torun, 1473 - Frombork, 1543)}

Nicolaj Kopernik, astrónomo de procedencia polaca, planteó un paradigma heliocéntrico del Universo, según el cual, el Sol se encuentra situado en el centro del Universo. Para el astrónomo establecido en Italia, la Tierra y los otros planetas se mueven sobre sí mismos y giran alrededor del Astro Solar en elipses circulares. En este paradigma, la Luna se mueve alrededor de la Tierra.

Copérnico se percató de la repercusión que sus ideas tendrían para las autoridades eclesiásticas. No obstante, en 1543 publicó su obra De Revolutionibus Orbium Coelestium (Las revoluciones de los cuerpos celestes).

Cuando publicó su libro De revolutionibus, lo recibió en su lecho de muerte (...) Los primeros en oponerse fueron los teólogos protestantes; la iglesia católica guardó discreto silencio (...) y sólo aceptó el sistema copernicano en 1820 (Azofeifa 1984: 177-8).

\subsection{Giordano Bruno (Nápoles, 1548 - Roma, 1600)}

Astrónomo italiano, pensaba que el Sol no era el centro del Universo, y que debía existir algo más allá del Sistema Solar. Giordano Bruno sufrió persecusión y fue llevado a la hoguera a causa de sus planteamientos.

\subsection{Galileo Galilei (Pisa, 1564 - Arcetri, 1642)}

Astrónomo italiano, construyó el telescopio que él mismo utilizó para contemplar el firmamento. Con su telescopio, Galileo observó que la Vía Láctea estaba constituida por millares de estrellas; descubrió manchas oscuras en el Sol, cráteres en la superficie de la luna, cuatro de las lunas de Júpiter, los anillos de Saturno y las fases de Venus.

La publicación de su libro Diálogo sobre los dos principales sistemas del mundo: el Copernicano y el Tolomeico, le causó un conflicto con la Iglesia.

No obstante, el aporte de Galileo fue de muchísima importancia, ya que sentó las bases de una nueva forma de investigación, sustentada en la observación y en la experimentación.

Para concluir este apartado, comparativamente hablando, podríamos decir que los astrónomos supra citados apuntaron el telescopio con la razón, mientras que Francisco de Asís lo apuntó con el corazón.

\section{Obra de Francisco de Asís}

\subsection{La Regla}

A través del testimonio de la historia y de los escritos que nos han llegado, la redacción latina de la Regla, en la que se manifiesta el propósito de Francisco de Asís, tomó aproximadamente una década. Un breve texto, conformado particularmente de citas evangélicas, fue presentado para la aprobación oral de Inoncencio III (1209 ó 1210). 
Dicho texto se fue ampliando con el crecimiento del grupo, que, al cabo de diez años pasó de doce a unos tres mil miembros. Este escrito, conformado por veintitrés capítulos, se conoce con el nombre de "Primera Regla". Las últimas capas de esta Regla datan de 1221.

Por diversos motivos (extensión del texto, falta de exactitud, presión de algunos miembros de la orden), Francisco de Asís se vio en la necesidad de asumir la elaboración de un texto más breve y condensado en doce capítulos, que, en 1223, recibió la aprobación de Honorio III. A este segundo texto se le conoce con el nombre de "Segunda Regla".

Tres años más tarde, cuando Francisco de Asís habla de la Regla en su Testamento, no hace ninguna diferencia de fondo: para él existe solamente una Regla, que manifiesta una sola intención. Según sus biógrafos, una lectura profunda y crítica de estas instrucciones fundamentales señala la permanencia de un proyecto idéntico: el amor fraterno dedicado a los pobres.

S. Francesco D'Assisi ritorna in Italia, si dedica alla riorganizzazione dell'ordine. Da papa Onorio III il 29 novembre 1223 ottiene il secondo riconoscimento della regola, nella quale con l'amore fraterno si esalta quello per la povertà. È il suggello della sua azione. umana. Nella chiesetta di S. Damiano il 1225 s'innalza il Cantico di Frate Sole. Siamo alla vigilia della morte (Acrosso 1955: 13, el destacado es nuestro).

\subsection{Cantico delle Creature}

Literariamente hablando, la obra de Francisco de Asís que interesa en este estudio es el Cantico delle Creature o Cántico di Frate Sole, cuya versión española del original italiano realizada por Manuel Durán, se encuentra dentro de las notas ${ }^{2}$. Los datos restantes de la fuente se encuentran consignados en la bibliografía de este artículo.

\section{Cantico delle Creature o Cantico di Frate Sole}

"ALTISSIMU, onnipotente, bon Signore, tue so' le laude, la gloria et l'honore et onne benedictione.

Ad te solo, Altissimo, se konfano et nullu homo ène dignu te mentovare.

Laudato sie, mi' Signore, cum tucte le tue creature, spetialmente messor lo frate sole,

lo qual'è iorno, et allumini noi per lui.

Et ellu è bellu e radiante cum grande splendore:

de te, Altissimo, porta significatione.

Laudato si', mi' Signore, per sora luna e le stelle: in celu l'ài formate clarite et pretiose et belle.

Laudato si', mi' Signore, per frate vento et per aere nubilo et sereno et onne tempo, per lo quale a le tue creature dài sostentamento.

Laudato si', mi' Signore, per sor'acqua, la quale è molto utile, et humele et pretiosa et casta.

Laudato si', mi' Signore, per frate focu, per lo quale ennallumini la nocte: ed ello è bello et iocundo et robustoso et forte. 
Laudato si', mi' Signore, per sora nostra matre terra,

la quale ne sustenta e governa,

et produce diversi frutti con coloriti fiori et herba.

Laudato si', mi' Signore, per quelli, ke perdonano per lo tuo amore

et sostengo infirmitate et tribulatione.

Beati quelli ke'l sosterranno in pace

ka da te, Altissimo, sirano incoronati.

Laudato si, mi Signore per sora nostra morte corporale,

da la quale nullu home vivente pò skappare:

guai a quelli ke morrano ne le peccata mortali;

beati quelli che trovarà ne le tue sanctissime voluntati,

ka la morte secunda no'l farà male.

Laudate e benedicete mi' Signore et rengratiate,

e serviateli cum grande humilitate".

(Getto, 1982: 16-17).

Según los estudiosos de la obra de Francisco de Asís, el Cántico de las Criaturas brotó de sus labios en un ambiente armonioso, luminosísimo y sereno, pese a los intensos dolores corporales por los que, en el momento de la creación lírica, transitaba el poeta.

En cuanto al tema tratado en el Cántico, la mayor parte de los críticos lo juzgan como inspiración del Salmo $148^{3}$ y de himnos de alabanza cantados en el Antiguo Testamento; y no faltan quienes comparen al autor del Cántico de las Criaturas con los poetas de la antigüedad clásica y con los provenzales y Minnesänger, amadores y cantores de las bellezas de los seres creados.

En lo concerniente al tema propiamente religioso, se desprende que así como los himnos laudatorios hebraicos están teñidos de fuerte emoción religiosa, el Cántico de las Criaturas es poesía que lleva impreso el sello místico de las alabanzas del Dios altísimo, pues quienes conocen la vida de Francisco de Asís, sabrán que las Sagradas Escrituras, a través de la liturgia cotidiana, moldearon su alma al igual que a estos salmistas.

Tuttavia anche se formalmente puro e conciso il Cantico di Frate Sole (1224) ha una sua ben definita ascendenza di cultura: la Sacra Scrittura (Getto 1981: 15).

Apuntan los biógrafos que Francisco de Asís amaba toda la creación, con afección especial al sol, pues para el poeta es el astro y la criatura más hermosa que más se asemeja al Creador, ya que Dios mismo se autodenomina en las Sagradas Escrituras: "Sol de justicia". El más antiguo de ellos, Tomás de Celano (1229), atestigua:

Llenábase de inefable gozo cuantas veces miraba el sol, o contemplaba la luna, o dirigía la vista a las estrellas y al firmamento (...) Finalmente, daba el dulce nombre de hermanas a todas las criaturas, de quienes, por modo maravilloso y de todos desconocido, adivinaba los secretos como quien goza ya de la libertad y la gloria de los hijos de Dios (in Boff 1982: 59). 
El Cántico de las Criaturas es un himno que brota espontáneamente del corazón de un asceta muy humano, enamorado de las bellezas de lo creado y por ello rebosante de gratitud a su Creador: quiere comunicar este agradecimiento a sus semejantes como medio de progreso y elevación hacia Dios.

L'ispirazione del Cantico è incentrata su di un motivo: l'umile eppure gioiosa accettazione di tutta la vita che palpita in noi e attorno a noi (...) provenendo da un Dio che è gioia suprema (...) bontà e bellezza che ci sono attestate da tutte le cose, l'acqua, il sole, la terra, il fuoco, i fiori, le stelle, armoniosamente disposti in questo grandioso universo (...) per essere utili alla nostra vita (Pazzaglia 1969: 104).

Francisco de Asís también gustaba mucho del canto, y logró que su Orden se reuniera para cantar alabanzas a Cristo y a la Virgen. Por esta razón, en este período, surgen los poetas llamados "laudesi", autores de himnos sagrados, a menudo en forma dialogada.

Le laude umbre hanno un tono popolaresco e immediato: tendono ad ispirare nel cuore sentimenti semplici e fervidi, soprattutto sul tema della passione di Cristo e del culto della Vergine (Pazzaglia 1969: 102).

En el siglo XIII surgen, pues, los primeros brotes de la lírica italiana: canciones populares de inspiración amorosa, política c religiosa.

El primer poeta italiano que compuso un himno de alta inspiración religiosa y en líricas de intención decididamente literaria en "romance vulgar" es Francisco de Asís.

Il primo documento letterario in volgare è il Cantico delle creature di san Francesco D'Assisi; esso è considerato la prima testimonianza di alto valore letterario, ma anche un caso unico, originale ed esemplare. La poesia scritta da Francesco è il prodotto più alto di un movimento religioso e culturale che ha un ruolo di primo piano nella formazione della lingua e della poesia in Italia. Il rapporto tra poesia e religione, tra impegno dell'autore e obiettivi di edificazione e di persuasione, è alla base della grande stagione letteraria del volgare (Ricciardi 1992: 48, el destacado es nuestro).

El mismo crítico literario expresa que el Cántico de las Criaturas, compuesto por Francisco de Asís en 1224, es:

Il primo grande componimento in lingua italiana scritto con piena coscienza e consapevolezza letteraria da parte dell'autore (1992: 50, el destacado es nuestro).

Giuseppe Petronio también afirma que:

San Francesco, oltre a varie opere minori, tra cui le Regole e il Testamento, fu autore di quel Canticus creaturarum o Cantico delle creature, tra le opere più antiche della nostra letteratura (fu composto un anno o due prima della morte del santo, il 1224-1225), in volgare umbro (1971: 42 , el destacado es nuestro).

Las diferentes citas textuales correspondientes a las opiniones vertidas por los estudiosos y los críticos literarios, en relación con el Cántico de las Criaturas, respaldan el contenido del presente artículo: por una parte, le atribuyen al poeta de Umbría la autoría del 
Cántico; por otra, también, le otorgan a este el debido mérito literario; y por último, coinciden en que el Cántico del Hermano Sol es el primer texto literario del Duecento escrito en "romance vulgar".

\section{Consideraciones finales}

La Italia del siglo XIII se caracteriza por un altísimo grado de religiosidad, cuya figura más importante es Francisco de Asís, uno de los poetas más destacados de la época, seguido por su discípulo Iacopone da Todi, autor de los Laudi, himnos de apasionado misticismo.

Vastos movimientos de religiosidad popular, promovidos por hombres que al soplo de lo divino unían las dotes de arrastrar a las gentes, se derraman desde la Umbría a las regiones limítrofes y después a toda Italia. Primero y más importante, el franciscanismo; luego, el movimiento del Alleluia (1223); después, el de los Flagelantes (1260) (Migliorini 1968: 202).

El Cántico de las Criaturas, esplendorosa obra maestra de Francisco de Asís, refleja el auténtico lirismo de su alma y un noble sentimiento hacia la Naturaleza: el poeta dejó profunda huella en la cultura europea y en la vida espiritual. La fortaleza de su carácter, su humildad y su profundo y fraternal amor a todas las criaturas, le conquistaron el aprecio y el reconocimiento popular.

Francisco de Asís descolló no solamente en el campo humano, como se demuestra a lo largo del presente artículo; el Cantico delle Creature logró marcar un hito como aporte a la historia de la lengua italiana, pues fue escrito en romance vulgar umbro y no en latín, como lo dictaba la norma.

Tal relevancia tiene dicha manifestación lírica, que todas las literaturas consultadas incluyen a Francisco de Asís como poeta importante del Duecento.

\section{Notas}

1. Según Cecchi y Sapegno (972: 634), Tomás de Celano retrata a San Francisco de Asís como se narra a continuación, en su "Legenda prima": "Facundissimus homo, facie hilaris, vultu benignus, immunis ignaviae, insolentiae expers. Statura mediocris, parvitati vicinior, caput mediocre ac rotundum, facies utcumque oblonga et protensa, frons plana et parva, mediocres oculi, nigri et simplices, fusci capilli, supercilia recta, nasus aequalis, subtilis et rectus, aures erectae sed parvae, tempora plana, lingua placabilis, ignea et acuta, vox vehemens, dulcis, clara atque sonora, dentes coniuncti, aequales et albi, modica labia atque subtilia, barba nigra, pilis non plene respersa, collum subtile, humeri recti, brevia brachia, tenues manus, digiti longi, ungues producti, crura sbtilia, parvuli pedes, tenuis cutis, caro paucissima, aspera vestis, somnus brevissimus, manus largissima".

2. Cántico de las Creaturas o Cántico del Hermano Sol: "ALTISIMO, omnipotente, buen Señor:/ Tuyas sean las alabanzas, la gloria y el honor, y todas la bendiciones./ A Ti solo, Altísimo, son debidas,/ y no hay hombre digno de mencionar Tu nombre./ Alabado seas, Señor, con todas Tus criaturas,/ y especialmente mi.señor el Hermano Sol,/ que nos da el día, y por quien Tú nos muestras la luz./ Es hermoso, y brilla con gran esplendor;/ de Ti, Altísimo, es símbolo./ Alabado seas, Señor, por la Hermana Luna y las estrellas;/ Tú las has formado en los cielos, claras, preciosas y bellas./ Alabado seas, Señor, por el Hermano Viento,/ y por 
VÍQUEZ: El Cántico de las Criaturas de Francisco de Asís: un aporte a la historia de la lengua... .

el aire y la nube por el buen y el mal tiempo, /con lo cual das sustento a Tus criaturas./ Alabado seas, Señor, por la Hermana Agua, /que es tan útil, humilde, preciosa y casta./ Alabado seas, Señor, por el Hermano Fuego, /gracias al cual iluminas la noche,/ y es hermoso y alegre, vigoroso y fuerte./ Alabado seas Señor, por nuestra Hermana Madre Tierra,/ que nos sustenta y nos da alimento,/ y produce frutos diversos, con brillantes flores y hierbas./ Alabado seas, Señor, por aquellos que por Tu amor perdonan,/ que soportan enfermedades y tribulación;/ bienaventurados los que lo soportan todo en paz,/ pues por Ti, Altísimo, serán coronados./ Alabado seas, Señor, por nuestra Hermana la Muerte del Cuerpo,/ de la cual ningún hombre vivo puede escapar;/ pobres de aquellos que mueren en pecado mortal,/ y benditos sean los que figuran en Tu santísima voluntad,/ pues la segunda muerte no les hará daño alguno./ Alabad, bendecid y dad gracias a mi Señor,/ y servidle con gran humildad".

3. Alabanza universal (Salmo 148): "AAleluya/ Alabad al Señor desde los cielos,/ Alabadlo en las alturas;/ alabadlo, todos sus ángeles;/ alabadlo, todos sus ejércitos;/ alabadlo, sol y luna,/ alabadlo, todas las estrellas luminosas; / alabadlo, cielos de los cielos/ y aguas que estáis por encima de los cielos; / alaben el nombre del Señor,/ porque él lo mandó y fueron creados;/ él se fijó para siempre jamás,/ puso unas leyes que nunca cambiarán./ Alabad al Señor desde la tierra,/ monstruos marinos y todos los abismos,/ fuego y granizo, nieve y bruma,/ viento de tempestad que ejecuta sus órdenes,/ montañas y todas las colinas,/ árboles frutales y todos los cedros,/ bestias salvajes y todos lo ganados,/ reptiles y pájaros que vuelan,/ reyes del mundo y pueblos todos, / príncipes y todos los jueces de la tierra, / jóvenes y todos los jueces de la tierra,/ jóvenes y también doncellas,/ los viejos a una con los niños;/ que todos alaben el nombre del Señor,/ porque su nombre es sublime, sólo él;/ su majestad domina los cielos y la tierra./ Él ha realzado el poder de su pueblo,/ orgullo para todos sus amigos,/ para Israel, su pueblo íntimo./ ¡Aleluya!” (La Santa Biblia. 1988. Madrid: Ediciones Paulinas, pág. 770).

\section{Bibliografía}

Acrosso, Paolo. 1955. Panorama: Antologia di Scrittori Italiani e Stranieri. Roma: Cappelli Editore.

Azofeifa, Isaac Felipe. 1984. Literatura Universal: Introducción a la Literatura Moderna de Occidente. San José: Editorial Universidad Estatal a Distancia.

Boff, Leonardo. 1982. San Francisco de Asís: ternura y vigor. Santander: Editorial "Sal Terrae".

Cecchi Emilio y Natalino Sapegno. 1972. Storia della Letteratura Italiana. Roma: Garzanti Editore.

Dante Alighieri. 1992. La Divina Comedia. Tercera reimpresión. México: Editores Mexicanos Unidos.

Durán, Manuel. 1961. Selección, versión y prólogo de la Antología de la Poesía Italiana (dirigida por Pablo González Casanova). México: UNAM, Dirección General de Publicaciones.

Getto, Giovanni y otros. 1982. Antología e Storia della Letteratura Italiana 1. III Edizione. Italia: Editrice La Scuola. 
Matura, Thaddée. 1978. El Proyecto Evangélico de Francisco de Asís Hoy. Madrid: Ediciones Paulinas.

Migliorini, Giuseppe. 1971. L'attività letteraria in Italia: Storia della letteratura. Italia: Palumbo.

Moreno, Jesús y Pedro Peira. 1979. Crestomatía románica medieval. Madrid: Ediciones Cátedra.

Pazzaglia, Mario. 1969. Gli Autori della letteratura italiana. Bologna: Zanichelli editore.

Petronio, Giuseppe. 1971. L'attività letteraria in Italia: Storia della letteratura. Italia: Palumbo.

Quirós Rodríguez, Manuel Antonio. 1997. "Inicio de la Universidad y de su terminología académica". Káñina: Revista de Artes y Letras de la Universidad de Costa Rica. 21(1): 13-187.

Robert, Paul. 1984. Petit Robert 2: Dictionnaire des noms propres. 8e édition. Paris: Le Robert.

Ricciardi, Mario. 1989. La Letteratura in Italia. Milano: Edizioni Acanthus.

Sapegno, Natalino. 1948. Disegno Storico della Letteratura Italiana. Firenze: La Nuova Italia.

Vidos, B.E. 1977. Manual de Lingüística Románica. Segunda edición. Madrid: Ediciones Aguilar.

Vossler, Karl. 1951. Historia de la Literatura Italiana. Segunda edición. España: Editorial Labor, S.A. 